\title{
A Stochastic Methodology for Predictions of the Environment Created by Multiple Microvibration Sources
}

\author{
M. Remedia ${ }^{\mathrm{a}}$, G.S. Aglietti ${ }^{\mathrm{a}}$, G. Richardson ${ }^{\mathrm{b}}$, M. Sweeting $^{\mathrm{b}}$ \\ ${ }^{a}$ Surrey Space Centre, University of Surrey, Guildford, GU2 7XH, UK \\ ${ }^{b}$ Surrey Satellite Technology Limited (SSTL), Guildford, GU2 7YE, UK
}

\begin{abstract}
It is well documented that at frequencies beyond the first few modes of a system, the Finite Element Method is unsuitable to obtain efficient predictions. In this paper, it is proposed to merge the efficiency of the Craig-Bampton reduction technique with the simplicity and reliability of Monte Carlo Simulations to produce an overall analysis methodology to evaluate the dynamic response of large structural assemblies in the mid frequency range. The method (Craig-Bampton Stochastic Method) will be described in this article with a benchmark example shown and implemented in the theory of the dynamic coupling extended to the case when multiple sources of microvibrations act simultaneously on the same structure. The methodology will then be applied to a real practical application involving the modern satellite SSTL 300 S1.
\end{abstract}

Keywords: Mid-Frequency, Dynamic Coupling, Craig-Bampton, Stochastic, Monte Carlo, Component Mode Synthesis

\section{List of Abbreviations}

$\mathrm{FE}=$ Finite Element

$\mathrm{SEA}=$ Statistical Energy Analysis

SFEM = Stochastic Finite Element Method

MCS $=$ Monte Carlo Simulation

CMS $=$ Component Mode Synthesis

$\mathrm{CB}=$ Craig-Bampton

CBSM = Craig-Bampton Stochastic Method 
$\mathrm{PSD}=$ Power Spectral Density

SQM = Structural Qualification Model

RSS $=$ Root Sum Square

\section{Introduction}

It is well known and documented that the Finite Element (FE) Method [1] gives accurate predictions for static analyses, and dynamics as long as the frequency does not exceed that of the first few structural modes of vibration $[2]$, that is where the behaviour of physical structures is quite deterministic. However, increasing the frequency the behaviour of real structures becomes more sensitive [3], and even nominally identical structures can produce relatively different high frequency responses (this is particularly evident for complex structural assemblies). In this high frequency range statistical approaches are more suitable, and here Statistical Energy Analysis [4, 5, 6] (SEA) has been applied quite successfully [7, 8, 9]. Between low frequency and high frequency (i.e. in the mid frequency) FE predictions start to become inefficient (good predictions could still be obtained, but building the appropriate detailed model would be extremely difficult and very demanding from a computational point of view), and SEA is not applicable as some of its basic assumptions are not yet validated.

The mid-frequency range is of particular interest in the study of spacecraft structures, and this work has been developed in the context of a project concerning analyses of transmission of microvibrations in satellites, but it is also applicable to many other fields, as long as the requirements described later on in the introduction are satisfied.

With the term microvibrations we generally refer to accelerations in the region of micro $g$, and generally these low level mechanical disturbances occur over a wide frequency range, from "zero" up to say 500-1000 Hz. In addition to the issue of their control and minimisation $[10,11,12]$, because of the large bandwidth of the frequency range, the modelling and analysis of microvibrations pose a challenge. This is a particular issue in the mid-frequency range as many of the micro-vibration sources on board a spacecraft (e.g. rotating mechanisms as reaction wheels, which are used to control the attitude of the satellite, or antenna pointing mechanisms) excite the structure in the mid-frequency range. In this case, besides the typical issues related to predicting responses in the mid-frequency, the low amplitude of the inputs can produce further uncertainties which can manifest themselves as nonlin- 
earities. A typical example is the behaviour of cables secured onto panels $[13,14,15]$ when very low forces are applied: the presence of the cables can influence the characteristics of the panel in terms of stiffness and damping values. The cables themselves become paths for vibration transmission and modelling them with simple non-structural mass (as it is often done for structural analyses) does not give accurate results.

Various approaches have been considered to solve micro-vibration analyses and mid-frequency problems in general. Many approaches can be found in MID-MOD [16]; here, finite element and statistical approaches (or both at the same time) are described. A literature review on a series of methods used to investigate the mid- frequency issue has been given by Desmet [2] One of the most successful implementations described in the book is the hybrid method developed by Shorter and Langley [17, 18]: the structure is divided into subsystems and some of them are described as finite element models, whilst others, which display a resonant behaviour, are studied with SEA. This method gives excellent results, providing the subsystems are appropriately selected. In the same context, also less recent works can be taken into account, such as Huiban and Baillion [19], who introduced the modal hybridisation. This method consists in building up responses given by the contributions of all the modes located in certain bands of the frequency spectrum. The drawback is that it strongly overestimates the response where modal overlap starts to be consistent.

One of the most classical types of approach used, is to extend the range of validity of the FE method results to higher frequencies and to account for uncertainties in parameters affecting the dynamic responses. This group of methods, which often consists of some pre or post processing the data obtained from a FE analysis, is called Stochastic Finite Element Method [20] (SFEM). One of the most well-known analysis types is the Monte Carlo Simulation (MCS), which is the simplest method for treating the response variability calculation in the framework of SFEM. Here, a number of samples of the stochastic system are generated (i.e. perturbing some of the structural parameters), and for each one of them the equilibrium equation is solved in order to evaluate the response leading to a population of the response vectors. MCS gives the best (most realistic) results, and in fact it is often used as a benchmark to compare the performance of other methods [21, 22], but it is still too computationally expensive, especially for large models of structures. A considerable amount of research has been published on approaches aimed at reducing the computational effort spent for the analysis, most of them 
using classical reduction methods [23]. The Craig-Bampton (CB) reduction method [24] has been chosen for the work described in this article, for various reasons: it is particularly suitable for base shake analysis, such as the analysis of satellite structures supported by the launch vehicle; and, most importantly, it is used for the Component Modal Synthesis (CMS) [25], which is one of the key techniques utilised in this paper. In addition, most FE models of specific satellite subsystems are delivered by subcontractors to the company that assembles the overall satellite structure in a reduced form (typically using CB reduction and therefore without giving any of the geometric and property details). For this reason it is difficult to implement a full MCS with perturbations of physical structural parameters for those specific components.

Amongst some of the proposed methodologies, Sarsri et al. [26] combined CMS and polynomial chaos basis to investigate the frequency transfer functions for large linear FE models of beams and assembled plates. A subspace iteration scheme has been developed by Pradlwarter et al. [27] to be used instead of CMS under specific circumstances. If uncertainties are in the joints [28] or in the boundaries [29] of the subsystems, CMS is the best approach for two reasons: the dynamic behaviour of the reduced structures is represented with just a few modal coordinates; the boundary degrees of freedom are still physical and therefore a MCS can still be applied using them. One of the most successful works developed in this context is the one published by Soize [30], which applies perturbations on the reduced matrices replacing the eigenvectors produced with mid-frequency energy eigenvectors.

In the context of robust dynamic condensation methods, a very interesting work has been developed by Guedri and Bouhaddi [31, 32]: here, the stochastic surface method and the non-dominated sorting genetic algorithm are coupled to take into account the propagation of uncertainties in FE models. In addition, two modal approaches have been presented by Van den Nieuwenhof and Coyette [33] as an alternative to direct formulations for dynamic analysis of structures with random material and shape parameters. Elsewhere, the dynamic condensation has been approached from an iterative perspective [34].

In this article we propose a variation of the CMS to merge the efficiency of CB reduction with the simplicity and reliability of MCSs for the various subsystems to produce an overall analysis methodology. This approach (which for sake of simplicity in this article will be called Craig-Bampton Stochastic Method, CBSM) is a good compromise between the simple mode superposi- 
tion, which produces results which are not reliable after the first few modes of vibration, and a computationally expensive full MCS. This method therefore succeeds in both reducing drastically the computational effort involved and proposing an approach which can be easily implemented by well-known FE method software. Mace and Shorter [35] have proposed the concept for a method that estimates frequency response function statistics in structures with uncertain parameters using an approach involving CMS. However, as the perturbations were limited to the stiffness matrix, it was not able to perturb the characteristics of each subsystems modes, which on the other hand the full MCS does. In this article we therefore extend the method including also variations in the mass matrix , which poses the complication of keeping the mass properties of the subsystems balanced throughout the process (i.e. the effective masses corresponding to the modal participation factors have to sum up to the total mass of the spacecraft after each randomisation), but gives the advantage of obtaining more reliable predictions, as it will be shown in section 2.3 .

In addition the paper illustrates a semi-empirical method to couple multiple sources of microvibrations (which are notoriously difficult to model appropriately $[36,37])$ with the spacecraft structure with the overall purpose to obtain predictions that envelope the actual response. The method also applies to non-microvibration implementations, but it is of particular interest in this field because of the mid-frequency being an issue when microvibration sources are acting.

The whole procedure on how to deal with microvibrations created by different sources acting on a structure has then been validated with an industrial application involving the modern satellite structure SSTL 300-S1, which carries a camera providing sub-metre imagery and is therefore important for microvibration studies. The satellite was made available by the company Surrey Satellite Technology Limited (SSTL) in Guildford, UK.

\section{Theory}

When a source of vibrations (reaction wheels, antenna pointing mechanisms etc) acts on a structure, there are different methodologies that can be applied to calculate the effect produced on the assembly.

The current practice in the spacecraft structures' industry is to measure the excitation forces created by the source when rigidly mounted (blocked) on a rigid multi-axis dynamometric platform (Kistler table) [38, 39], and then ap- 
ply them to the location where the source is mounted on the structure, adding a lumped inertia which represents the source [40, 41, 42, 43]. This method clearly gives good results if the source's structure has resonance frequencies well beyond the frequency range of interest. In the case of microvibration this is not possible, and therefore the dynamics of the source need to be considered, as illustrated in the next section.

\subsection{Dynamic Coupling for 1 source}

As the sources are dynamically coupled to the satellite $[43,44,38]$, in order to determine their impact on the platform response, two aspects have to be considered:

- The passive effect of the body (or structure) of the source itself. E.g., even when a reaction wheel is switched off, its presence will affect the dynamics of the satellite;

- The active phenomena within the source which produce mechanical loads during operation of the specific piece of equipment, e.g. the mass unbalance of a flywheel which produces a centrifugal force when the rotor is spinning, or the mechanical noise produced by the bearings.

The passive effect can be reproduced using a detailed FE model of the source (note that to produce accurate FE models of rotating mechanisms such as reaction wheels or antenna pointing mechanisms is relatively challenging) or using experimental results.

Concerning the active phenomena that generate the loads within the source, modelling from first principle phenomena (e.g. noise produced by the bearings) is relatively challenging. The simpler approach is to use an experimental method, and directly measure the blocked reaction loads with the source on a dynamometer.

Therefore the objective here is to reproduce the whole effect of the source with just an equivalent input force, which takes into account also the dynamic coupling of the source with the supporting structure. This permits to avoid having to build a FE model of the source, as its dynamic effect can be induced using the source dynamic mass which can be directly measured.

The loads produced by the vibrating equipment (source) on its supporting structure depend on the dynamic characteristics of the source itself and those of the supporting structure. Thus, intuitively, it can be said that a portion of the force that the source generated in its blocked configuration $\left(\mathbf{f}_{\mathbf{B}}\right)$ will 
now be used to move the source itself (as its mounting interface has acceleration different from zero). Therefore the force $\mathbf{f}_{\mathbf{C}}$ that the source can actually transmit to the coupled supporting structure will be:

$$
\mathbf{f}_{\mathrm{C}}=\mathbf{f}_{\mathrm{B}}-\mathbf{M}_{\mathrm{S}} \ddot{\mathbf{x}}
$$

Where $\mathbf{M}_{\mathbf{S}}$ is the frequency dependent dynamic mass of the source and $\ddot{\mathbf{x}}$ is the acceleration at the interface point. The underlying assumption here is that the source is connected to the satellite structure through a single interface node with six degrees of freedom. Hence the vectors $\mathbf{f}_{\mathbf{C}}, \mathbf{f}_{\mathbf{B}}$ and $\ddot{\mathbf{x}}$ have six frequency dependent elements, whereas $\mathbf{M}_{\mathbf{S}}$ is the 6 x6 dynamic mass matrix which contains the reaction loads produced by the source when unit accelerations along the interface degrees of freedom are imposed.

In order to determine $\ddot{\mathbf{x}}$ it is necessary to have some knowledge of the supporting structure, and in particular its response at the driving point (e.g. the driving point accelerance $\mathbf{A}_{\text {str }}$, or its inverse, the dynamic mass $\mathbf{M}_{\text {str }}$ of the satellite at the location of the source):

$$
\ddot{\mathbf{x}}=\mathbf{A}_{\text {str }} \mathbf{f}_{\mathrm{C}}
$$

As the acceleration at the interface appears in Eq.(1), it is possible to substitute Eq.(2) into Eq.(1) to obtain:

$$
f_{C}=f_{B}-M_{S} A_{s t r} f_{C}
$$

Which can be solved for $\mathbf{f}_{\mathbf{C}}$ to yield:

$$
\mathbf{f}_{\mathbf{C}}=\left(\mathbf{I}+\mathbf{M}_{\mathbf{S}} \mathbf{A}_{\mathbf{s t r}}\right)^{-1} \mathbf{f}_{\mathbf{B}}
$$

Eq.(4) shows the difference that actually exists between the blocked forces (measured on a Kistler table) and the coupled forces (measured when the source is physically mounted on the satellite). An increase of the dynamic mass values corresponds to a lower value of the force that is actually transmitted to the spacecraft compared to the forces produced in a blocked configuration. Eq.(4) can also be re-written using random vibrations parameters. The blocked forces can be described by the Power Spectral Density (PSD) matrix of the reaction forces and moments produced by the source in its blocked configuration $\boldsymbol{\Phi}_{\mathbf{B}}$. The term in bracket in Eq.(4) is the transfer function between $\mathbf{f}_{\mathrm{B}}$ and $\mathbf{f}_{\mathbf{C}}$. In random vibration analysis PSDs are utilised; therefore, in order to obtain the PSD of the output $\boldsymbol{\Phi}_{\mathbf{C}}$, the PSD of the 
input $\boldsymbol{\Phi}_{\mathbf{B}}$ needs to be pre-multiplied by the matrix of the transfer function and post-multiplied by the Hermitian of the matrix of the transfer function:

$$
\boldsymbol{\Phi}_{\mathbf{C}}=\left(\mathbf{I}+\mathbf{M}_{\mathbf{S}} \mathbf{A}_{\mathbf{s t r}}\right)^{-1} \boldsymbol{\Phi}_{\mathbf{B}}\left(\mathbf{I}+\mathbf{M}_{\mathbf{S}} \mathbf{A}_{\mathbf{s t r}}\right)^{-\mathrm{H}}
$$

Where -H denotes the Hermitian operation. The PSD matrix at an arbitrary location on the spacecraft structures can then be obtained by multiplying by a transfer function matrix $\widetilde{\mathbf{T F}}_{\mathbf{C B}}$ :

$$
\Phi_{\mathbf{O}}=\widetilde{\mathbf{T F}}_{\mathbf{C B}}\left(\mathbf{I}+\mathbf{M}_{\mathbf{S}} \widetilde{\mathbf{A}}_{\mathbf{s t r}}\right)^{-1} \boldsymbol{\Phi}_{\mathbf{B}}\left(\mathbf{I}+\mathbf{M}_{\mathbf{S}} \widetilde{\mathbf{A}}_{\mathbf{s t r}}\right)^{-\mathrm{H}} \widetilde{\mathbf{T F}}_{\mathbf{C B}}^{\mathrm{H}}
$$

Where $\widetilde{\mathbf{T F}}_{\mathbf{C B}}$ will be calculated through an implementation using the method that will be described in section 2.3 in order to take into account the uncertainties related to the FE model of the structure. The accent $\sim$ is used to represent the terms that will be perturbed.

\subsection{Dynamic Coupling for more than 1 source}

When more than one source of vibrations is involved as input, the coupling (Eq.(6)) can still be applied, but the matrices will be larger in order to consider the degrees of freedom of the interfaces of the various systems. As a term of reference, a generic problem is represented in Figure 1.

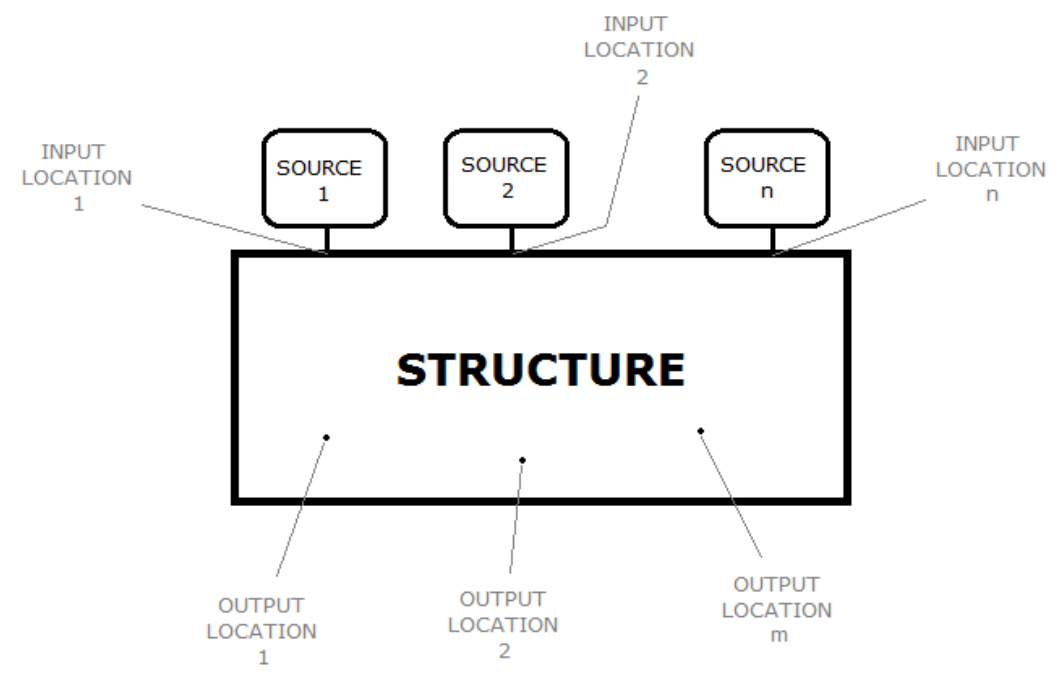

Figure 1: Schematic representation of a generic structure 
Assuming $n$ sources which are physically separated (whose effects are connected only through the supporting satellite structure), the overall dynamic mass of the sources will be composed by the $6 \mathrm{x} 6$ dynamic mass matrices of the various sources, positioned on the diagonal of the overall dynamic mass matrix:

$$
\mathbf{M}_{\mathbf{S}}=\left(\begin{array}{cccc}
\mathbf{M}_{\mathrm{S} 1} & \mathbf{0} & \cdots & \mathbf{0} \\
\mathbf{0} & \mathbf{M}_{\mathrm{S} 2} & \cdots & 0 \\
\vdots & \vdots & \ddots & \vdots \\
0 & 0 & \cdots & \mathbf{M}_{\mathrm{Sn}}
\end{array}\right)
$$

However, the accelerance matrix of the satellite structure will be a fully populated matrix because all the sources' interface degrees of freedom are affected by the inputs along any of the interface degrees of freedom:

$$
\mathbf{A}_{\text {str }}=\left(\begin{array}{cccc}
\mathbf{A}_{\text {str11 }} & \mathbf{A}_{\text {str12 }} & \cdots & \mathbf{A}_{\text {str1n }} \\
\mathbf{A}_{\text {str21 }} & \mathbf{A}_{\text {str22 }} & \cdots & \mathbf{A}_{\text {str2n }} \\
\vdots & \vdots & \ddots & \vdots \\
\mathbf{A}_{\text {strn1 }} & \mathbf{A}_{\text {strn2 }} & \cdots & \mathbf{A}_{\text {strnn }}
\end{array}\right)
$$

The $6 \times 6$ blocks on the diagonal of the overall accelerance matrix are the driving point accelerances calculated at the interfaces of the sources and the off diagonal blocks represent the interactions between the sources produced by the supporting satellite structure.

The overall force PSD matrix of the blocked sources has a structure similar to that of the accelerance, where the off diagonal blocks would represent cross-correlations between different sources.:

$$
\Phi_{\mathrm{B}}=\left(\begin{array}{cccc}
\Phi_{\mathrm{B}_{11}} & \boldsymbol{\Phi}_{\mathrm{B}_{12}} & \cdots & \boldsymbol{\Phi}_{\mathrm{B}_{1 \mathrm{n}}} \\
\boldsymbol{\Phi}_{\mathrm{B}_{21}} & \boldsymbol{\Phi}_{\mathrm{B}_{22}} & \cdots & \boldsymbol{\Phi}_{\mathrm{B}_{2 \mathrm{n}}} \\
\vdots & \vdots & \ddots & \vdots \\
\Phi_{\mathrm{B}_{\mathrm{n} 1}} & \boldsymbol{\Phi}_{\mathrm{B}_{\mathrm{n} 2}} & \cdots & \boldsymbol{\Phi}_{\mathrm{B}_{\mathrm{nn}}}
\end{array}\right)
$$

Finally $\widetilde{\mathbf{T F}}_{\mathbf{C B}}$ (see Eq.(6)) represents the matrix of the transfer functions between the input(s) and the output(s) locations. If $m$ is the number of output locations considered, $\widetilde{\mathbf{T F}}_{\mathbf{C B}}$ will have a $m x 6 n$ size. Also this matrix is a full matrix as potentially all inputs can affect all outputs.

In conclusion, Eq.(6) for the case when more than one source is acting simultaneously on the same structure can still be used, but all the matrices apart 
from $\widetilde{\mathbf{T F}}_{\mathbf{C B}}$ and $\boldsymbol{\Phi}_{\mathbf{O}}$ now have size $6 n x 6 n$.

A numerical example where two different sources are utilised is shown in Appendix A.

The last step left to perform before applying this method is to determine the transfer function matrix $\widetilde{\mathbf{T F}}_{C B}$ and the satellite structure accelerance. This will be done in the next section taking into account the uncertainties associated with the satellite structural dynamics discussed in the introduction.

\subsection{Craig-Bampton Stochastic Method}

With the implementation of the theory of the coupling, all the sources can be replaced by inputs corrected considering the dynamic behaviour of the sources themselves and the structure they act on. In order to do so, the accelerance of the structure needs to be computed, together with the transfer functions between the input and the output locations.

These parameters are of difficult computation, especially when considering spacecraft as structures (which FE models are relatively complicated, hence very easily affected by modelling inaccuracies). In order to overcome this issue, a MCS is generally applied to the structure, but in this article the substructuring approach taken so far (used when the structure was separated from the sources of vibrations) will be extended. In particular a method, which takes advantage of the component mode synthesis (often necessary to be used when companies provide their products only as reduced matrices) implementing a simplified MCS on it, will be used. The same predictions computed by a full MCS can be produced, but with a fraction of the computational effort.

The method is based on an integration of the components' FE models reduced with CB, which is particularly applicable in the space sector as the spacecraft structures' components are often delivered by subcontractors in the form of $\mathrm{CB}$ reduced models.

For convenience of the reader, the main steps of the CB reduction are summarised below (for more details see reference [45]). The equation governing the dynamics of the whole systems can be written as:

$$
\mathbf{M} \ddot{\mathbf{x}}+\mathbf{C} \dot{\mathbf{x}}+\mathbf{K x}=\mathbf{f}
$$

Where $\mathbf{M}, \mathbf{C}$ and $\mathbf{K}$ are respectively the mass, damping and stiffness matrices of the structure, $\mathbf{x}$ is the vector of the physical degrees of freedom and $\mathbf{f}$ is the vector of the forces. 
The structure can be divided in subsystems $i$ and the degrees of freedom in each subsystem can be divided in two different groups: $R$ (boundary degrees of freedom, which also include the desired input and output degrees of freedom) and $L$ (other degrees of freedom):

$$
\mathrm{x}_{\mathrm{i}}=\left(\begin{array}{c}
\mathrm{x}_{\mathrm{iR}} \\
\mathrm{x}_{\mathrm{iL}}
\end{array}\right)
$$

As an example, the structure of Figure 1 has been divided into 4 subsystems (see Figure 2). Here, all the $\mathrm{R}$ degrees of freedom are represented with a bigger circle: the boundary points connecting the various subsystems, the input locations where the sources act and the desired output locations.

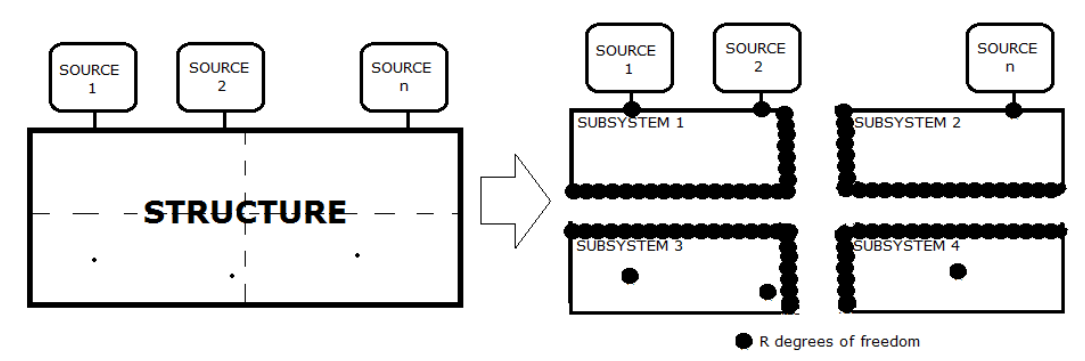

Figure 2: Division of a generic structure into subsystems for CB reduction

The $L$ degrees of freedom can then be converted into modal degrees of freedom with the advantage of choosing the number of modal coordinates to consider for the analysis. As the response can be reconstructed using only the modes which give significant contributions the number of modal coordinates is significantly smaller than the number of physical degrees of freedom of the subsystem.

The transformation matrix can therefore be set as:

$$
\mathrm{x}_{\mathrm{i}}=\left(\begin{array}{c}
\mathrm{x}_{\mathrm{iR}} \\
\mathrm{x}_{\mathrm{iL}}
\end{array}\right)=\left(\begin{array}{cc}
\mathrm{I} & 0 \\
\phi_{\mathrm{iR}} & \phi_{\mathrm{iL}}
\end{array}\right)\left(\begin{array}{l}
\mathrm{x}_{\mathrm{iR}} \\
\mathrm{q}_{\mathrm{im}}
\end{array}\right)=\Gamma\left(\begin{array}{c}
\mathrm{x}_{\mathrm{iR}} \\
\mathrm{q}_{\mathrm{im}}
\end{array}\right)
$$

Where $\phi_{\mathbf{i} \mathbf{R}}$ is a transformation matrix that relates rigid body physical displacements at the interface to physical displacements of the elastic degrees of freedom, $\mathbf{q}_{\mathbf{i m}}$ are the modal responses, $\phi_{\mathbf{i L}}$ is a transformation matrix that relates the modal responses qim $_{\text {im }}$ to physical displacements of the elastic degrees of freedom, $\boldsymbol{\Gamma}$ is the Craig-Bampton transformation matrix, $\phi_{\mathbf{i R}} \mathbf{x}_{\mathbf{i R}}$ are 
the constrained node displacements and $\phi_{\mathbf{i L}} \mathbf{q}_{\mathbf{i m}}$ are the normal mode displacements.

Substituting these new coordinates into the equation of motion and premultiplying by the transpose of $\boldsymbol{\Gamma}$, it yields:

$$
\begin{aligned}
& \left(\begin{array}{ll}
\mathrm{M}_{\mathrm{iBB}} & \mathrm{M}_{\mathrm{iBm}} \\
\mathrm{M}_{\mathrm{imB}} & \mathrm{M}_{\mathrm{imm}}
\end{array}\right)\left(\begin{array}{c}
\ddot{\mathrm{x}}_{\mathrm{iR}} \\
\ddot{\mathrm{q}}_{\mathrm{im}}
\end{array}\right)+\left(\begin{array}{ll}
\mathrm{C}_{\mathrm{iBB}} & \mathrm{C}_{\mathrm{iBm}} \\
\mathrm{C}_{\mathrm{imB}} & \mathrm{C}_{\mathrm{imm}}
\end{array}\right)\left(\begin{array}{c}
\dot{\mathrm{x}}_{\mathrm{iR}} \\
\dot{\mathrm{q}}_{\mathrm{im}}
\end{array}\right)+ \\
& +\left(\begin{array}{ll}
\mathbf{K}_{\mathrm{iBB}} & \mathbf{K}_{\mathrm{iBm}} \\
\mathbf{K}_{\mathrm{imB}} & \mathbf{K}_{\mathbf{i m m}}
\end{array}\right)\left(\begin{array}{c}
\mathbf{x}_{\mathrm{iR}} \\
\mathbf{q}_{\mathrm{im}}
\end{array}\right)=\left(\begin{array}{c}
\mathbf{f}_{\mathrm{iR}}+\phi_{\mathbf{R}} \mathbf{T}_{\mathbf{i L}} \\
\phi_{\mathbf{R}} \mathbf{T}_{\mathbf{i L}}
\end{array}\right)
\end{aligned}
$$

Where, for mass normalised matrices, $\mathbf{M}_{\mathbf{i m m}}$ is an identitiy matrix, $\mathbf{M}_{\mathbf{i B B}}$ is the structural mass matrix reduced to the boundary nodes, $\mathbf{M}_{\mathbf{i B m}}$ is the matrix of the modal participation factors and $\mathbf{M}_{\mathbf{i m B}}$ is the transpose of $\mathbf{M}_{\mathbf{i B m}}$. $\mathbf{C}_{\mathbf{i B B}}, \mathbf{C}_{\mathbf{i B m}}$ and $\mathbf{C}_{\mathbf{i m B}}$ are all equal to 0; $\mathbf{C}_{\mathbf{i m m}}$ contains the modal damping values for each one of the modal coordinates considered; $\mathbf{K}_{\mathbf{i B m}}$ and $\mathbf{K}_{\mathbf{i m B}}$ are also equal to 0 and $\mathbf{K}_{\mathbf{i m m}}$ contains the natural frequencies of the subsystems with the boundary degrees of freedom constrained.

Substituting the terms described above in Eq.(13), the mass, damping and stiffness matrices become:

$$
\mathbf{M}_{\mathbf{i}}=\left(\begin{array}{cc}
\mathbf{M}_{\mathrm{iBB}} & \widetilde{\mathbf{M}}_{\mathrm{iBm}} \\
\widetilde{\mathbf{M}}_{\mathrm{imB}} & \mathbf{I}
\end{array}\right) \quad \mathbf{C}_{\mathbf{i}}=\left(\begin{array}{cc}
\mathbf{0} & \mathbf{0} \\
\mathbf{0} & 2 \xi \widetilde{\omega}_{\mathrm{i} 0}
\end{array}\right) \quad \mathbf{K}_{\mathbf{i}}=\left(\begin{array}{cc}
\mathbf{K}_{\mathrm{iBB}} & 0 \\
\mathbf{0} & \widetilde{\omega}_{\mathrm{i} 0}^{2}
\end{array}\right)
$$

Where the accent identifies the terms which will be perturbed.

In practice if the $\mathrm{CB}$ reduction is used for each of the various subsystems, the final overall model can be assembled from the various CB reduced models. Here, the subsystems are perturbed before their integration, and what was found was that perturbations of only resonance frequencies (as previously done by Mace and Shorter [35]) of the subsystems were not sufficient to capture the whole range of changes produced by the full MCS. The concept of extending the perturbation to the modal participation factors (in addition to the one of the natural frequencies) will be clearer in the benchmark example that follows.

All the reduced and perturbed subsystems can now be integrated back to form the stiffness and mass matrices of the whole structure and this can be solved. What is obtained is a perturbation of the aforementioned matrices which will give a very similar prediction to the MCS one, as will also be proven with the practical application in section 3 . 


\subsubsection{Benchmark Example Application}

As a benchmark example, a simple model including four beam elements will be considered (see Figure 3). All the beam elements are $0.5 \mathrm{~m}$ long, with a Young's modulus of $6.9 \times 10^{10} \mathrm{~N} \mathrm{~mm}^{-2}$, a shear modulus of $2.55 \times 10^{10} \mathrm{~N} \mathrm{~mm}^{-2}$ and a density of $2800 \mathrm{~kg} \mathrm{~m}^{-3}$.

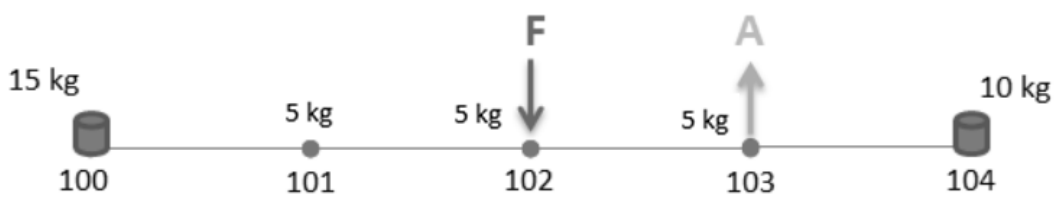

Figure 3: Schematic representation of the benchmark example

At the beginning of the first beam element and at the end of the last one masses of, respectively, 15 and $10 \mathrm{~kg}$ are attached, whilst in all the beam elements' connections there are masses of $5 \mathrm{~kg}$. For this example, the transfer function between node number 102 and node number 103 will be considered; therefore, a force equal to $1 \mathrm{~N}$ is applied to node 102 and the acceleration in node 103 is obtained as a response.

As the final solution is obtained using a mode superposition approach anyway, it is conceivable to solve the overall nominal problem and calculate the response using a mode superposition applying perturbations directly to the following equation:

$$
H=\sum_{i=1}^{n} H_{i}=\sum_{i=1}^{n} \frac{\widetilde{\varphi}_{i j} \widetilde{\varphi}_{i k}}{\widetilde{\omega}_{0 i}^{2}-\omega^{2}+2 \mathrm{j} \xi \widetilde{\omega}_{0 i} \omega}\left(-\omega^{2}\right)
$$

$\widetilde{\varphi}_{i j}$ and $\widetilde{\varphi}_{i k}$ are the nodal values of the eigenvectors of the force and the response respectively, $\widetilde{\omega}_{0 i}$ are the natural frequencies and $\xi$ is the damping (the damping value has been assumed here to be the same for all the natural frequencies). This simple procedure has been carried out and the results are shown in Figure 4. In order to obtain the modal perturbation prediction, a flat randomisation of $50 \%$ has been applied to the values in Eq.(15).

100 different computations have been performed and the final prediction is given by:

$$
H_{M}=\bar{H}+2 \sqrt{\frac{1}{N} \sum_{j=1}^{N}\left(H_{j}-\bar{H}\right)^{2}} \quad \text { with } \quad \bar{H}=\frac{1}{N} \sum_{j=1}^{N} H_{j}
$$


Where $H_{j}$ are all the $\mathrm{N}$ transfer functions computed.

Eq.(16) has been chosen for the final prediction because, among all the results that have been computed during the randomisation process, we want an envelope with a very high probability for the real result to be included. $2 \sigma$ gives a $95 \%$ probability (starting from the prerequisite that the real results is one of the 100 that have been built), which is sensible for our purposes.

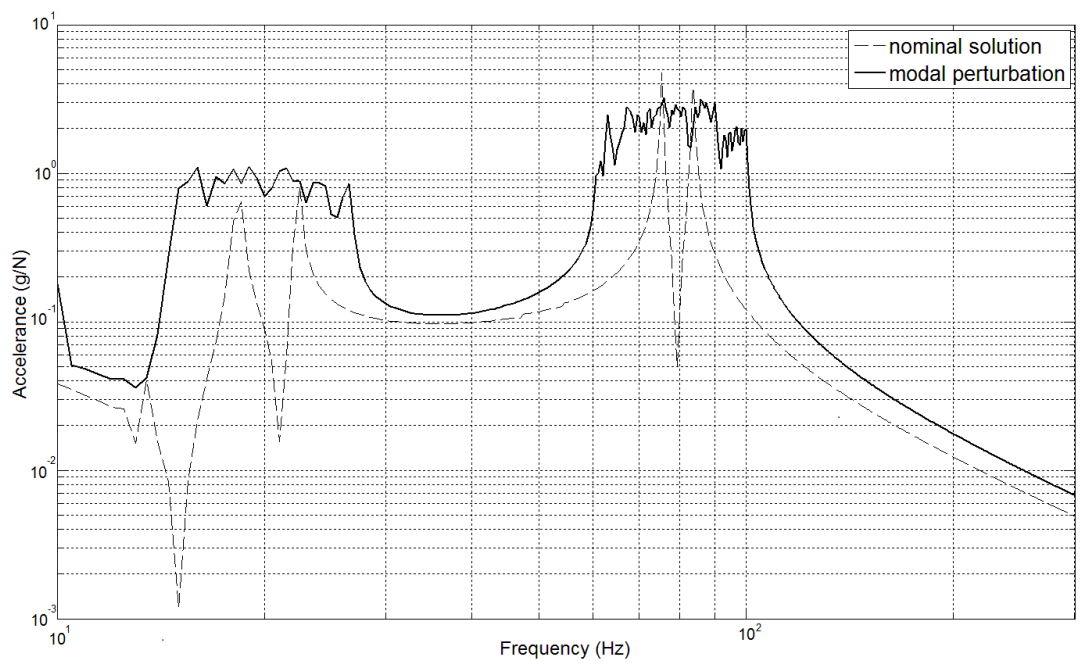

Figure 4: Perturbation of Natural Frequencies to the benchmark example

As a term of comparison, the full MCS is here applied, in order to obtain the desired transfer function. The Young's modulus and the density of the materials of most of the beam elements have been randomised. A flat probability density distribution of the randomisation has been chosen, giving the physical parameters in a range between a minimum and a maximum value (this is preferable to a Gaussian perturbation, which could also produce tail values too high or too low to be realistic for the system studied). In particular, the Young's modulus has been varied between $-5 \%$ and $+5 \%$, and the density between $-2.5 \%$ and $+2.5 \%$. 100 different models have been built and the final MCS prediction, obtained using Eq.(16), is shown in Figure 5a. Very important, here the reader should note that two new features, the peaks at 46 and $53 \mathrm{~Hz}$, have appeared in the response. These dynamics weren't depicted when applying a simple mode superposition (and also when frequencies were perturbed) because the outputs belonged to a nodal line of the mode shapes corresponding to the two frequencies. Perturbing some physical 
parameters changed the mode shapes, resulting in the two frequencies to be now showing a response when the input is applied in node 102. This effect cannot be reproduced simply perturbing a mode superposition.

Next is the implementation of the CBSM. First, the system is divided into two different subsystems and a residual structure, as shown in Figure 6.

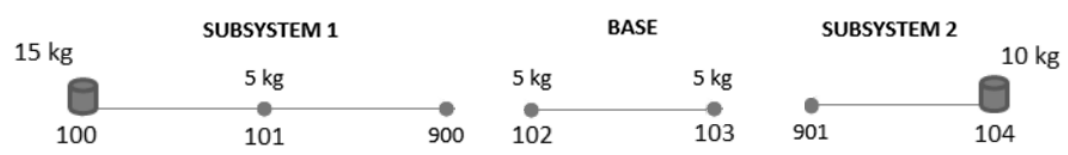

Figure 6: System divided into a residual structure and two subsystems

Subsystem 1 and Subsystem 2 are reduced with CB and their respective stiffness and mass matrices are obtained as outputs. The stiffness matrices contain the values of their natural frequencies with the boundary points constrained. These frequencies are perturbed with a Gaussian randomisation of $2 \sigma$ value equal to $10 \%$ and then the new matrices are integrated into the residual structure. The process has been repeated 100 times and the same statistical computations performed for the full MCS have been worked out also for the CBSM to produce the graph in Figure 5b.

Figure 5b shows the two new modes already predicted by the full MCS, though with a lower amplitude. Also in this case a change of the mode shapes of the whole structure was produced when perturbing the frequencies of the single subsystems. In order to enhance the effects produced by the perturbation of the natural frequencies of the various subsystems, the same perturbation of the modal participation factors in the reduced mass matrices has been associated. The new prediction is now shown in Figure $5 \mathrm{c}$.

Figure $5 \mathrm{c}$ shows again the two new peaks at 46 and $53 \mathrm{~Hz}$. In addition to that, also the effects of the new resonance frequencies over the whole frequency range are predicted, matching very closely what the full MCS predicted (Figure 5a). For instance, the perturbation of only the resonance frequencies didn't change at all the areas where there were no structural modes; this is compensated by the perturbation of the modal participation factors (the effect is clearly visible over $100 \mathrm{~Hz}$, but also on the various predicted peaks, which now assume a shape more similar to the full MCS). The main advantage of using the CBSM instead of the full MCS is the computational effort; as running models with the subsystems reduced with $\mathrm{CB}$ takes much less time than running the whole model. To prove the viability 


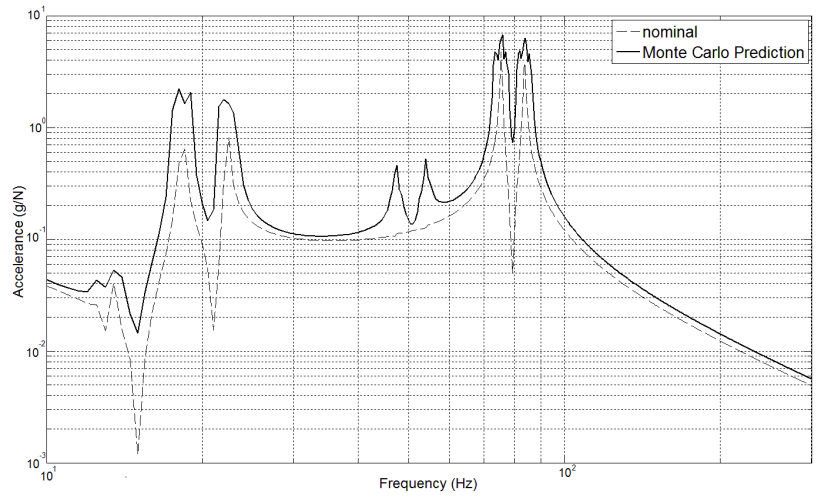

(a) Monte Carlo Simulation

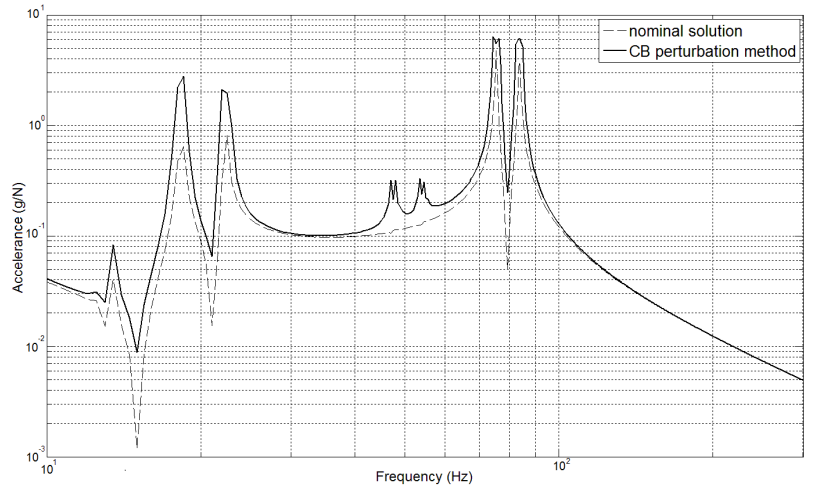

(b) Craig-Bampton Stochastic Method (frequencies)

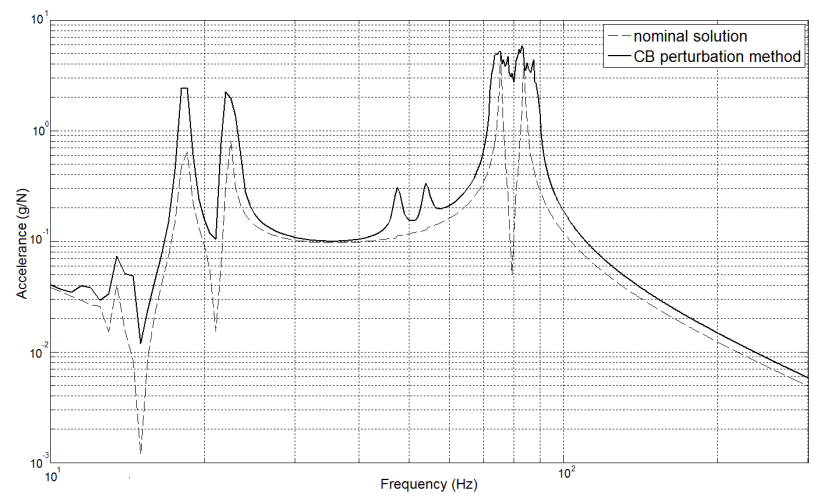

(c) Craig-Bampton Stochastic Method (frequencies and modal participation factors)

Figure 5: Analyses performed on the benchmark example 
of this method beyond the simple benchmark example just presented, in section 3 an application involving the satellite SSTL 300-S1 will be described and the results shown using both full MCS and CBSM.

\section{Industrial Application}

The methodology is now applied to a real spacecraft structure. The SSTL 300 S1 (see Figure 7) is the latest in the family of highly capable SSTL platforms, providing sub-metre imagery. Its Structural Qualification Model (SQM, i.e. a structure with the same properties as the real one built specifically for testing purposes) will be used here considering as sources of microvibrations one of its reaction wheels and one of its antenna pointing mechanisms.

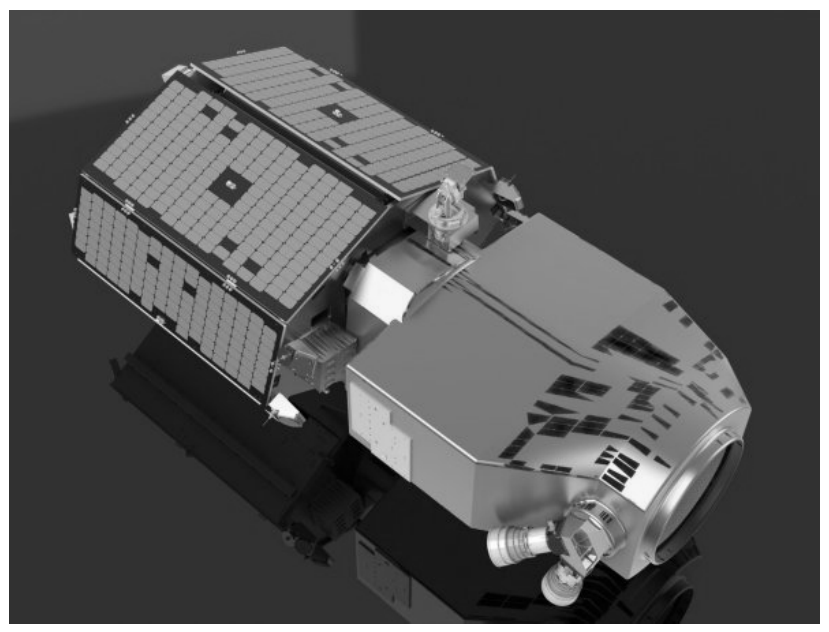

Figure 7: Satellite SSTL 300 S1

\subsection{Experimental Campaigns}

Four different test campaigns were carried out:

- The first one was on the spacecraft free in space to determine the transfer functions between sources and receivers and the satellite structure accelerance in order to validate the CBSM (test campaign 1).

- A second test campaign followed on the sources on Kistler table to work out the blocked forces (test campaign 2). 
- The aim of the third one was the measurement of the sources' dynamic masses (test campaign 3).

- The final test campaign consisted in retrieving the response of the satellite to the sources (integrated on the satellite) in order to verify that the whole methodology was able to envelop the results obtained by the test campaign (test campaign 4).

In test campaign 1, the SQM of the satellite SSTL 300 S1 has been hung with elastic cords to reproduce "free in space" conditions (Figure 8) and a first set of tests was conducted with the use of mini-shakers to reproduce unit forces at the input locations (Figure 9). Accelerations have been acquired on several locations of interest. The measured transfer functions have here been used as a term of reference for the implementation of MCS and CBSM.

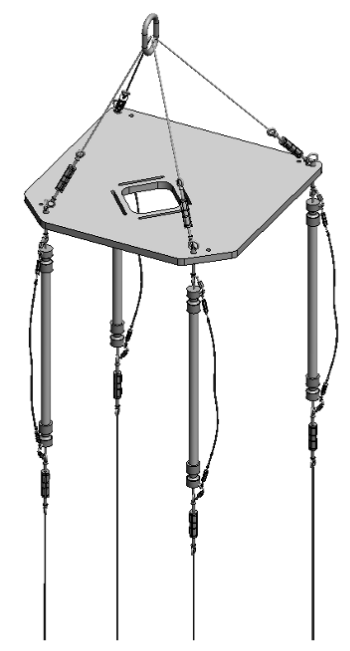

Figure 8: Suspension system to reproduce "free in space" conditions 


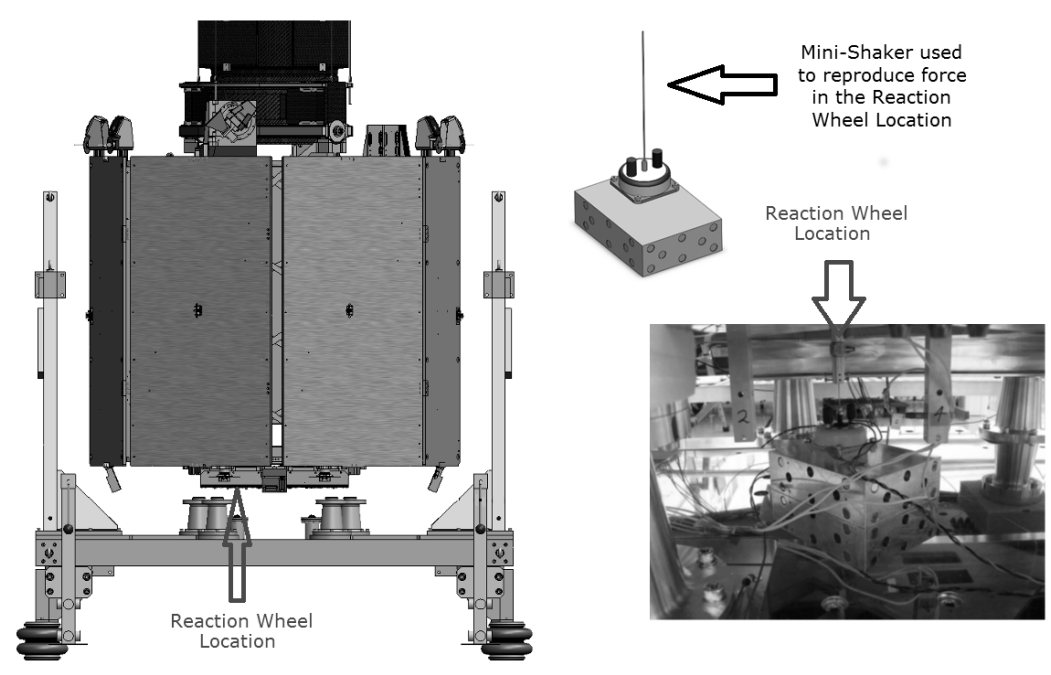

Figure 9: Experimental setup for mini-shaker testing on Reaction Wheel location

As for test campaign 2, Figure 10 shows one of the sources of microvibrations (reaction wheel) blocked on a Kistler Table. After analytical computations, the blocked forces in all the six degrees of freedom could be retrieved. The same has been done for the antenna pointing mechanism.

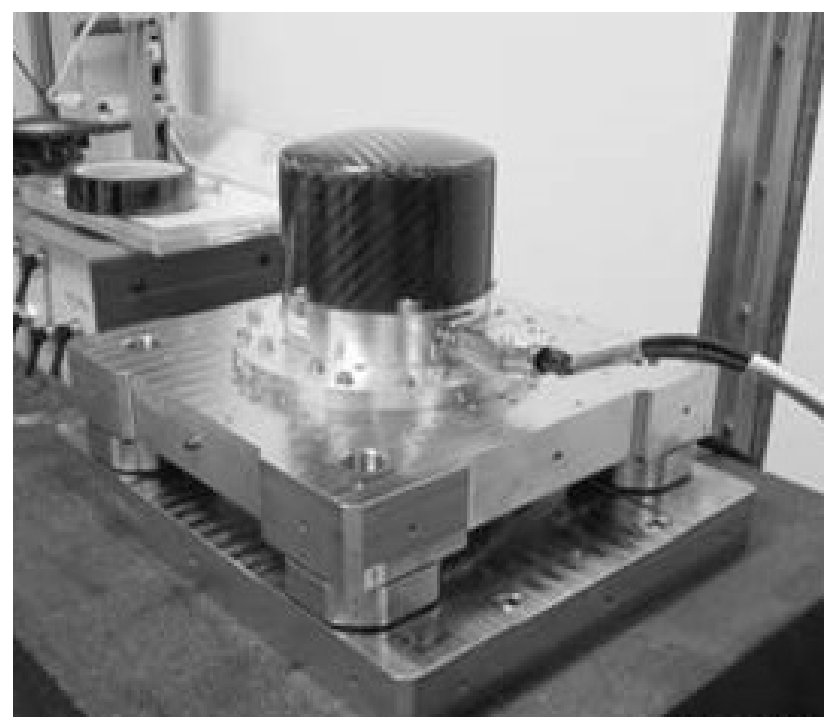

Figure 10: Measurement of the blocked forces 
In order to apply the theory of the coupling, the dynamic mass of the sources of microvibrations needed to be measured experimentally (preferred to computing it with the FE method, which can result in many inaccuracies). Pictures of the dynamic mass testing (test campaign 3) both for the reaction wheel and the antenna pointing mechanism are shown in Figure 11 and Figure 12, respectively.
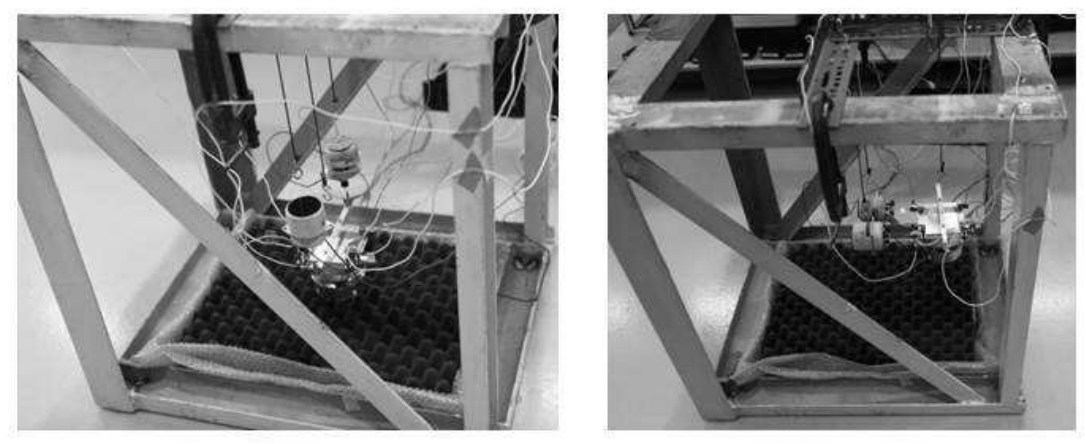

Figure 11: Reaction Wheel Dynamic Mass measurement
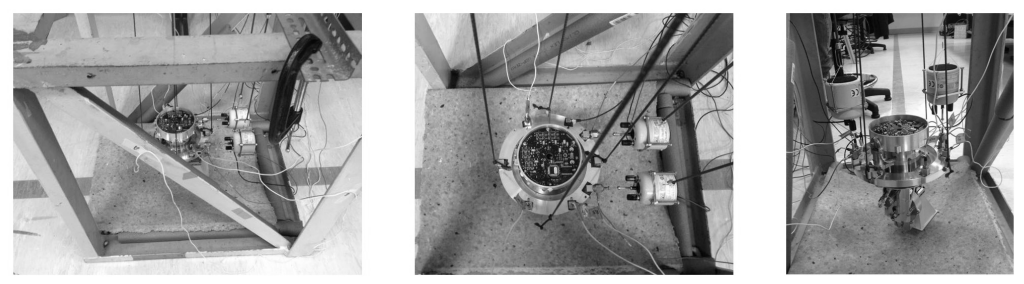

Figure 12: Antenna Pointing Mechanism Dynamic Mass measurement

Finally, in test campaign 4, real sources of microvibrations have been placed in the SQM of the satellite and run at specific velocities. First, the reaction wheel has been run at steps of $60 \mathrm{rpm}$ (Figure 13) and then the antenna pointing mechanism at four different velocities (Figure 14). Finally, both the sources have been acting contemporaneously on the spacecraft. The latter is the configuration that will be considered for our analyses in the next section. 


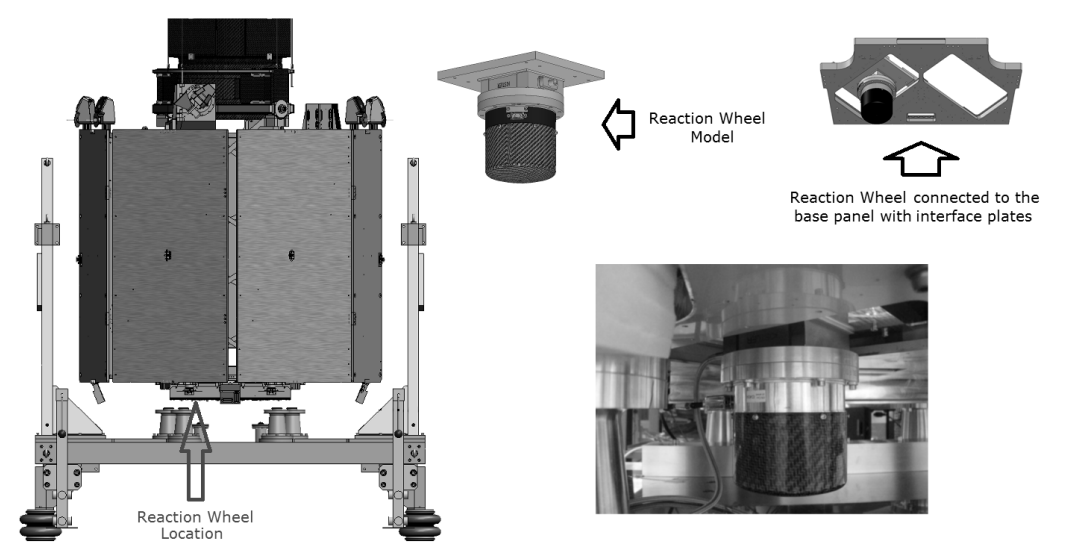

Figure 13: Experimental setup for reaction wheel testing

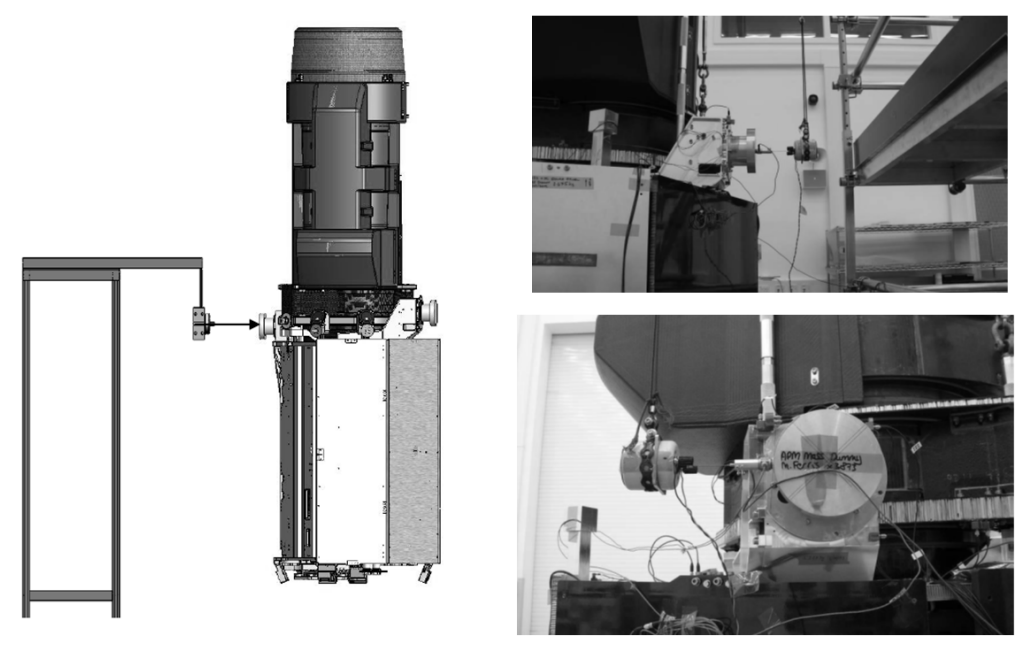

Figure 14: Experimental setup for antenna pointing mechanism testing

\subsection{Analyses}

The analyses presented in this article will be divided in two parts. Firstly, the transfer functions between the source's (Reaction Wheel or Antenna Pointing Mechanism) and the receiver's (any of the accelerometers' locations chosen for the experimental campaign) locations will be taken into account and the CBSM will be validated against a full MCS. Secondly, the procedure of the coupling for multiple sources (both Reaction Wheel and Antenna 
Pointing Mechanism acting simultaneously) will be applied to the satellite SSTL 300 S1: the results will be compared to the ones obtained applying the theory separately for each source. Both the predictions will be validated against experimental results.

\subsubsection{CBSM validation}

The FE model of the satellite without the sources of microvibrations in it has been considered for this analysis and it is shown in Figure 15.

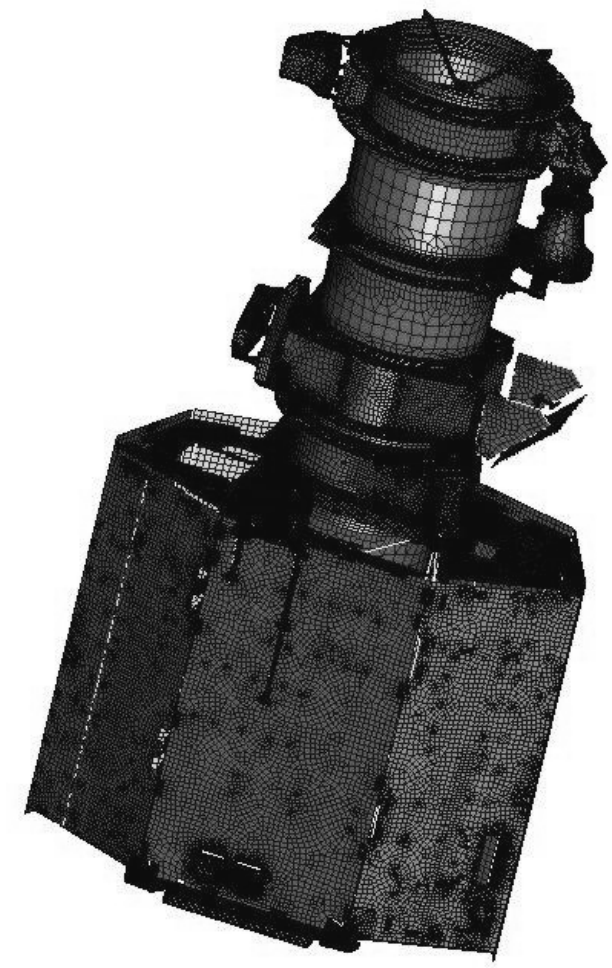

Figure 15: Finite Element Model of the satellite SSTL 300 S1

First, a full MCS has been applied. Gaussian perturbations (with $\sigma$ value of $5 \%$ ) of the main materials of the structure, of the stiffness of the joints and of the materials have been applied; 100 different models have been run and a final stochastic model has been built using Eq.(16).

For what concerns the implementation of the CBSM, it has been simulated that: 
- 2 components have been sent as reduced models from external companies;

- the panel where one of the sources of microvibrations will be placed has been modelled with a high level of uncertainty.

As a consequence, five different subsystems of the satellite have been created and they are shown in Figure 16.

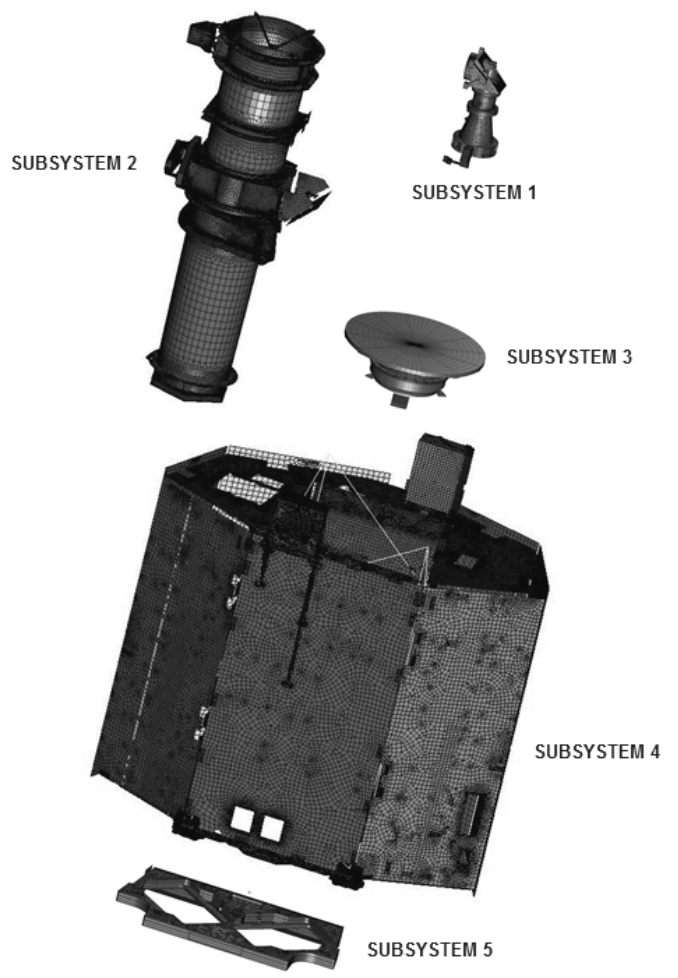

Figure 16: Subsystems of the FE model of the satellite SSTL 300 S1

In particular, subsystems 1 and 3 would be the ones provided by other companies (their FE models are only represented here for a better understanding of the procedure); subsystem 5 is the panel where the reaction wheel will act as source of microvibrations; subsystem 2 is the satellite platform and subsystem 4 is the payload.

All the subsystems have then been reduced with the $\mathrm{CB}$ reduction leaving the boundary points as physical degrees of freedom and for each subsystem 
computing a number of modal coordinates to be used for further analyses. Figure 17 shows a schematic representation of how the new satellite looks after the reduction. Each box represents a subsystem and the number of modal coordinates considered is indicated representing the boundaries kept as physical coordinates.

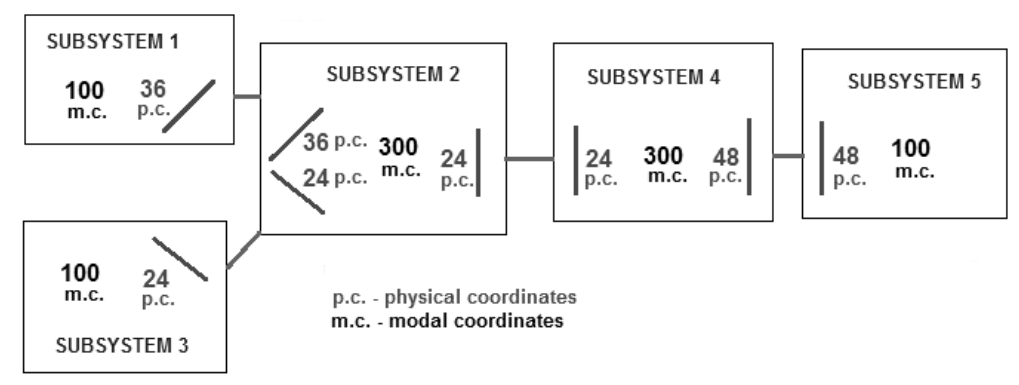

Figure 17: Schematic representation of the reduced subsystems

The number of modal coordinates also corresponds to the number of sets of modal participation factors included in the reduced mass matrices. These values and the natural frequencies (included in the stiffness matrices, see Eq.(14)) have been perturbed according to the level of uncertainty of the corresponding physical subsystems. This way, 100 different models have been built. They have all been run (with a minimal computational effort, compared to the one needed to compute 100 runs of entire satellite versions) and Eq.(16) has again been applied to obtain the stochastic model obtained through the implementation of the CBSM.

A full MCS has also been performed on the satellite. A Gaussian randomisation (with $2 \sigma$ value equal to $5 \%$ ) has been applied to the stiffness of the materials, the stiffness of the joints and the smeared mass representing harness and brackets. 100 different models have been built. Tranfer functions have been computed and again Eq.(16) has been used to obtain the final prediction.

The graph in Figure 18 shows the comparison between the predictions obtained using the stochastic models built with the MCS and the CBSM, together with the nominal solution for one of the output locations. As can be seen, in the mid-frequency range the predictions are very close to each other and given that the MCS method produces the best results for this kind of problem, this validates the use of the CBSM, which saves a considerable 
amount of time.

Looking at Eq.(6), the procedure to compute the values with the tilde on them is now known. In the next section the full methodology when a reaction wheel and an antenna pointing mechanism are used to create microvibrations is implemented.

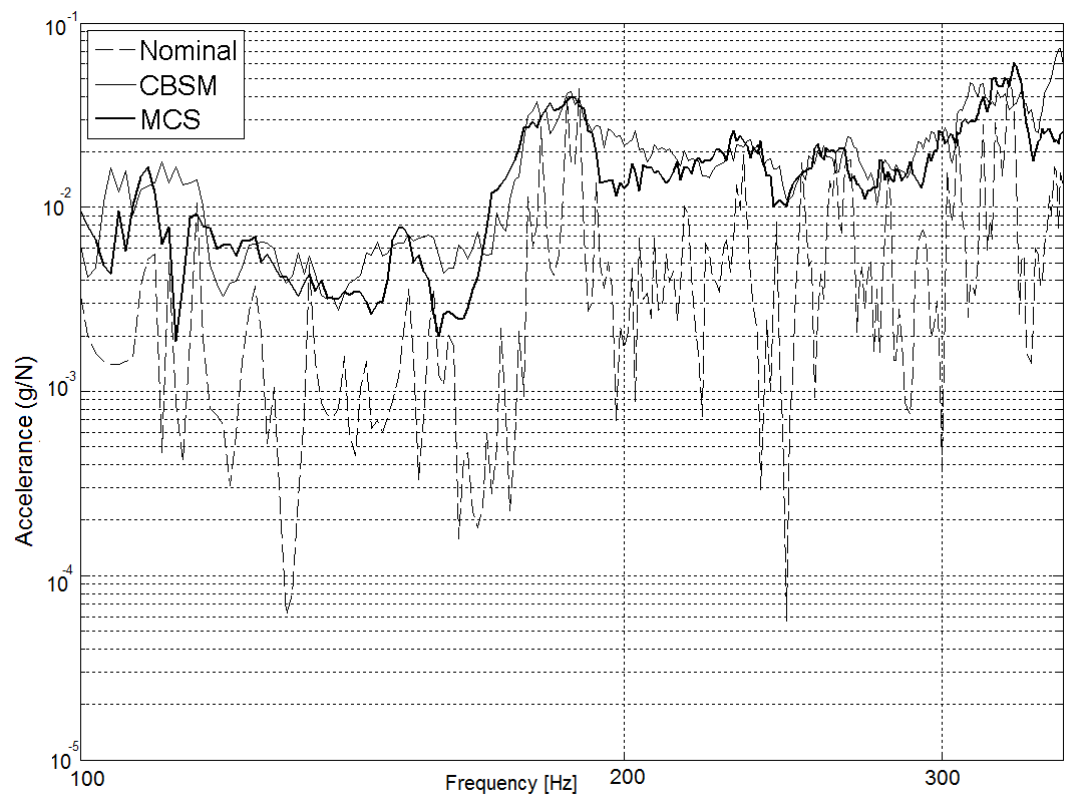

Figure 18: Full MCS and CBSM predictions compared to nominal solution

\subsubsection{Implementation Methodology}

From Eq.(6), $\widetilde{\mathbf{T F}}_{\mathbf{C B}}$ and $\widetilde{\mathbf{A}}_{\text {str }}$ can be computed following the procedure described in Section 3.2.1. $\mathbf{M}_{\mathbf{S}}$ is the matrix of the dynamic masses and it has been obtained experimentally with the testing shown in Figure 11 and Figure 12. Finally, $\boldsymbol{\Phi}_{\mathbf{B}}$ is the matrix of the inputs created by the sources of microvibrations in blocked configuration. Theoretically, the matrix $\mathbf{\Phi}_{\mathbf{B}}$ should be built also with all the non-off diagonal terms, but, as is shown with a benchmark example in Appendix A, integrating them into the computation causes only a negligible difference in the final output and therefore they are not included in the computation.

The computation of the same output considered for the CBSM validation has been done in two different ways:

- the reaction wheel and the antenna pointing mechanism have been 
modelled as lumped masses. The response in one specific output location has been calculated both for the case with the reaction wheel as input (i.e. the blocked forces of the reaction wheel have been inputted to the reaction wheel location) and for the case with the antenna pointing mechanism as input. The two responses have then been summed with a Root Sum Square (RSS, i.e. for each frequency step the root of the sum of the squared values of the two response at the same frequency step has been calculated);

- the theory of the coupling for multiple sources has been directly applied to the structure with both the sources in action.

The comparison between the two predictions is shown in Figure 19. The experimental result is also shown as a term for reference.

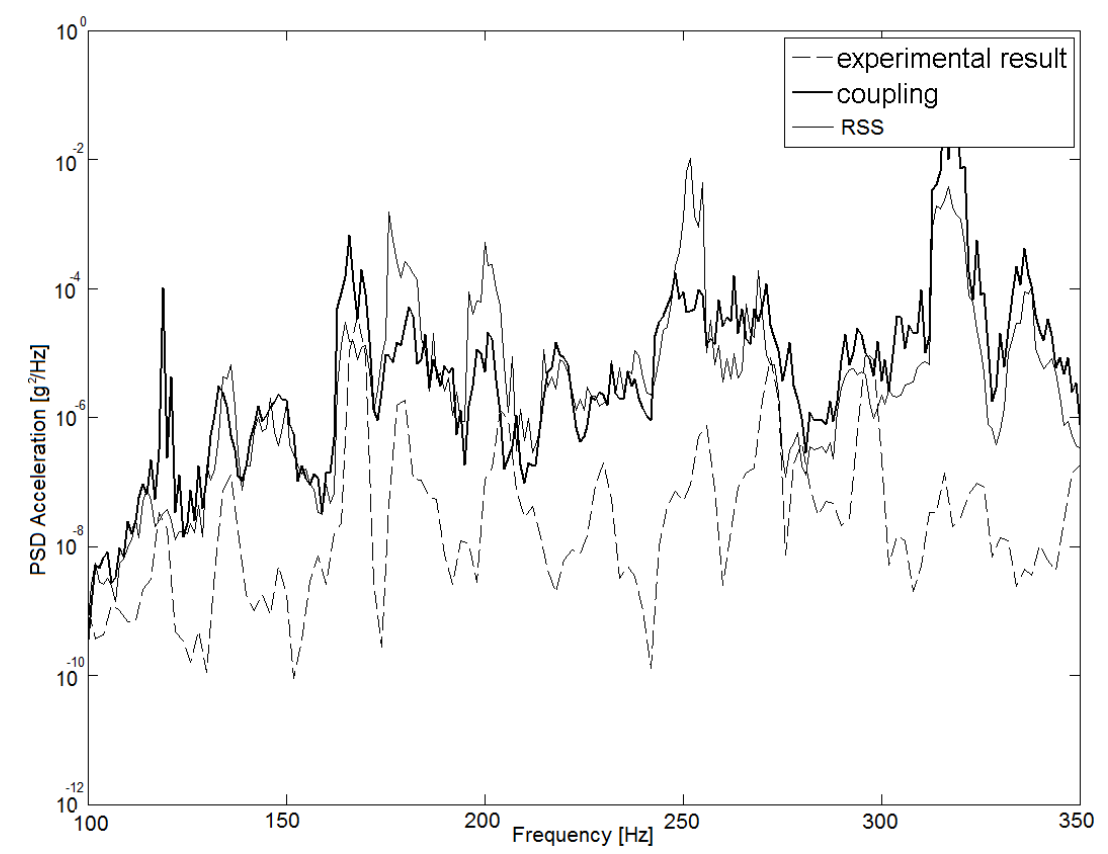

Figure 19: Predictions computed applying the theory of the coupling for multiple sources acting simultaneously on the same structure and leaving the sources as lumped masses and the responses computed through a RSS

All the predictions have been obtained using Eq.(16) when implementing the CBSM. An envelope is therefore created and the aim is to obtain a prediction that safely includes the real results, at cost of sometimes significantly 
overestimating the prediction. This happens also in the graph in Figure 19: some of the overestimations are actually "corrected" by the implementation of the theory of the coupling (see 160, 180 and $250 \mathrm{~Hz}$ ), others aren't (see $320 \mathrm{~Hz}$ ). The point that here the reader should observe is that, as proven in Appendix A, the theory of the coupling for multiple sources is an exact theory; calculating the response with a RSS is one of the common practices and Figure 19 shows how far this can be from the exact solution.

\section{Conclusions}

In this article, the viability of a stochastic method implementing CraigBampton reductions to predict the vibration response of a satellite structural assembly has been investigated. The main features of the method proposed have been demonstrated using a benchmark example, which has shown the capability to reproduce results very similar to those obtained from a full Monte Carlo Simulation, but with a fraction of computational effort. Besides the reduced computational effort required, this methodology also offers the possibility to implement different levels of uncertainties in various subsystems, which is particularly valuable in the space industry, as different parts of the satellite usually have different levels of maturity, characterised by different levels of uncertainty in their parameters.

The method has been implemented into a methodology aimed at computing predictions when multiple sources of microvibrations act simultaneously on the same structure.

The practical viability of the methodology has then been proved showing the application of this method to predict the micro-vibration environment for a realistic structural assembly, the satellite SSTL 300 S1. Two different sources of microvibrations have been used: a Reaction Wheel and an Antenna Pointing Mechanism. Results have shown improvements compared to the classical methods normally used and satisfyingly envelope the experimental results obtained after a testing campaign also described in this article.

\section{Acknowledgments}

The authors would like to thank the European Space Agency (and in particular Gregory Laduree) with the ESA project "Methodology for Analysis of Structure-Borne Micro-Vibration" for making this work possible. 


\section{Appendix A. Off-Diagonal Terms Evaluation and Multiple Sources Coupling Implementation}

In this appendix a benchmark example with two sources of vibrations and a receiver will be considered, both for the validation of the coupling theory for more than one source of vibrations and also for evaluating the importance of the computation of the off-diagonal terms of the input matrix when calculating the response acceleration PSD in an arbitrary point of a structure. Firstly, the system in Figure A.20a is considered. The sources and the receiver that will be used for the computation are circled.

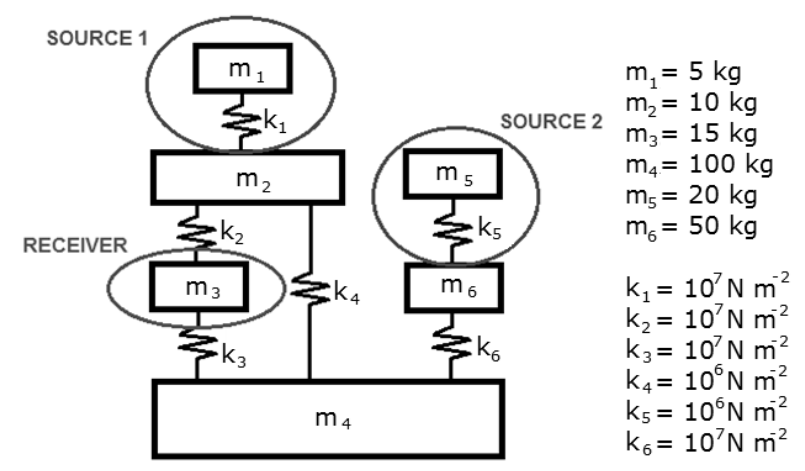

(a) Benchmark example with sources and receiver

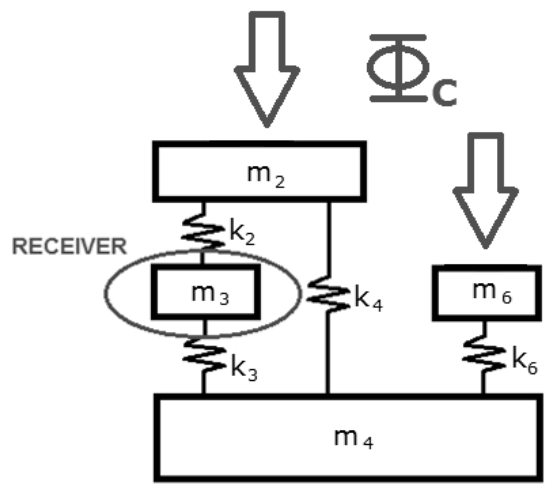

(b) Benchmark example with sources replaced by coupled forces

Figure A.20: Benchmark Example

The two sources produce forces which can be measured experimentally, 
for instance on a Kistler Table, and here it is assumed to produce a white noise. The aim is to compute the coupled forces $\boldsymbol{\Phi}_{\mathbf{C}}$ (Figure A.20b) and use them to produce the final output. This should be the same as the output predicted applying the blocked forces directly to the sources in Figure A.20a. The mass and stiffness matrices of the whole system can be written as:

$$
\begin{gathered}
\mathbf{M}=\left(\begin{array}{cccccc}
5 & 0 & 0 & 0 & 0 & 0 \\
0 & 10 & 0 & 0 & 0 & 0 \\
0 & 0 & 15 & 0 & 0 & 0 \\
0 & 0 & 0 & 100 & 0 & 0 \\
0 & 0 & 0 & 0 & 20 & 0 \\
0 & 0 & 0 & 0 & 0 & 50
\end{array}\right) \\
\mathbf{K}=\left(\begin{array}{cccccc}
10^{7} & -10^{7} & 0 & 0 & 0 & 0 \\
-10^{7} & 2.1 \cdot 10^{7} & -10^{7} & -10^{6} & 0 & 0 \\
0 & -10^{7} & 2 \cdot 10^{7} & -10^{7} & 0 & 0 \\
0 & 10^{6} & -10^{7} & 2.1 \cdot 10^{7} & 0 & -10^{7} \\
0 & 0 & 0 & 0 & 10^{6} & -10^{6} \\
0 & 0 & 0 & 10^{7} & -10^{6} & 1.1 \cdot 10^{7}
\end{array}\right)
\end{gathered}
$$

The sources can be separated from the rest of the structure. This yields:

$$
\begin{aligned}
& \mathbf{M}_{\mathbf{s t}}=\left(\begin{array}{cccc}
10 & 0 & 0 & 0 \\
0 & 15 & 0 & 0 \\
0 & 0 & 100 & 0 \\
0 & 0 & 0 & 50
\end{array}\right) \\
& \mathbf{K}_{\text {st }}=\left(\begin{array}{cccc}
2.1 \cdot 10^{7} & -10^{7} & -10^{6} & 0 \\
-10^{7} & 2 \cdot 10^{7} & -10^{7} & 0 \\
10^{6} & -10^{7} & 2.1 \cdot 10^{7} & -10^{7} \\
0 & 0 & 10^{7} & 1.1 \cdot 10^{7}
\end{array}\right) \mathbf{M}_{\mathbf{w} \mathbf{1}}=\left(\begin{array}{cc}
5 & 0 \\
0 & 0
\end{array}\right) \\
& \mathbf{K}_{\mathbf{w} \mathbf{1}}=\left(\begin{array}{cc}
10^{7} & -10^{7} \\
-10^{7} & 10^{7}
\end{array}\right) \mathbf{M}_{\mathbf{w} \mathbf{2}}=\left(\begin{array}{cc}
20 & 0 \\
0 & 0
\end{array}\right) \mathbf{K}_{\mathbf{w} \mathbf{2}}=\left(\begin{array}{cc}
10^{6} & -10^{6} \\
-10^{6} & 10^{6}
\end{array}\right)
\end{aligned}
$$

The dynamic mass matrix of the sources $\mathbf{M}_{\mathbf{S}}$ is given by:

$$
\mathbf{M}_{\mathbf{S}}=\left(\begin{array}{cc}
\mathbf{M}_{\mathbf{S 1}} & 0 \\
0 & \mathbf{M}_{\mathbf{S} 2}
\end{array}\right)
$$

This is because the dynamic masses are measured on the sources when they are disconnected from the structure and therefore there is no interaction 
between them. Hence the 0 in the off-diagonal terms. $\mathbf{M}_{\mathbf{S 1}}$ and $\mathbf{M}_{\mathbf{S 2}}$ can be calculated as follows:

$$
\begin{aligned}
& \mathbf{M}_{\mathbf{S} \mathbf{1}}=\left[-\mathbf{M}_{\mathbf{w} \mathbf{1}} \omega^{2}+\mathbf{K}_{\mathbf{w} \mathbf{1}}(1+0.02 \mathbf{j})\right]^{-1} \omega^{2} \\
& \mathbf{M}_{\mathbf{S} \mathbf{2}}=\left[-\mathbf{M}_{\mathbf{w} \mathbf{2}} \omega^{2}+\mathbf{K}_{\mathbf{w} \mathbf{2}}(1+0.02 \mathrm{j})\right]^{-1} \omega^{2}
\end{aligned}
$$

$\omega$ is the frequency. The accelerance (i.e. inverse of the dynamic mass) of the structure $\mathbf{A}_{\text {str }}$ is calculated with respect to the two points where the sources act. This time, the off-diagonal terms are not 0 since the two points belong to the same structure when is either tested or computed.

$$
\mathbf{A}_{\mathrm{str}}=\left(\begin{array}{ll}
\mathbf{A}_{\mathrm{str} 11} & \mathbf{A}_{\mathrm{str} 12} \\
\mathbf{A}_{\mathrm{str} 21} & \mathbf{A}_{\mathrm{str} 22}
\end{array}\right)
$$

The global input is given by:

$$
\mathbf{f}_{\mathbf{B}}=\left(\begin{array}{ll}
f_{B 11} & f_{B 12} \\
f_{B 21} & f_{B 22}
\end{array}\right)
$$

Where $f_{B 11}$ and $f_{B 22}$ are the PSDs of the forces produced by, respectively, source one and source two, $f_{B 12}$ is the Cross PSD of the forces of the two different sources, and $f_{B 21}=f_{B 12}$.

The new inputs now created considering the effect of the dynamic coupling between the sources and the rest of the structure can be computed using Eq.(4) (i.e. the foces corrected to include the dynamic interaction source/structure). The result is the black curve in Figure A.21. It can be verified that it is possible to obtain the same output response by applying simultaneously the inputs to the total model of the structure:

$$
\mathrm{OUT}=\mathbf{T F} \cdot \mathbf{I N P} \cdot \mathbf{T F}^{\mathrm{H}}
$$

Where OUT is the output in the receiver location, INP is the matrix of the forces applied (corrected to include the effect of the coupling) to the structure and $\mathbf{T F}$ is the matrix of the transfer functions from the input locations to the output location for the overall structure including the sources. 


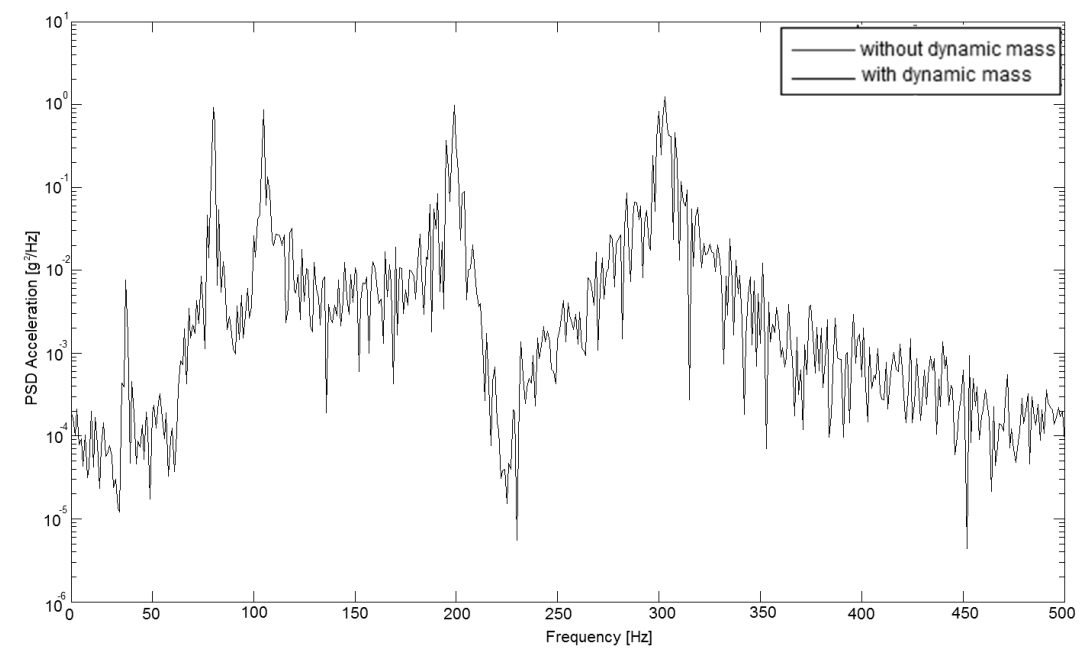

Figure A.21: Predictions with the source implemented in the model and the source included in the new dynamic input - note that the curves are identical

Figure A.21 shows that as expected the two computations give exactly the same result.

Next we are going to illustrate the effect of the cross terms in the PSD input force matrix. Two options can be considered now:

- Case A: neglect the off-diagonal terms, which means that only the PSDs of the signal are to be computed.

- Case B: calculate the PSDs and the Cross PSDs to build the whole input matrix.

This is how the input matrix looks like for the two cases:

$$
\Phi_{\mathrm{B}_{\mathrm{A}}}=\left(\begin{array}{cc}
\Phi_{\mathrm{B}_{11}} & 0 \\
0 & \Phi_{\mathrm{B}_{22}}
\end{array}\right) \quad \Phi_{\mathrm{B}_{\mathrm{B}}}=\left(\begin{array}{ll}
\Phi_{\mathrm{B}_{11}} & \Phi_{\mathrm{B}_{12}} \\
\Phi_{\mathrm{B}_{21}} & \Phi_{\mathrm{B}_{22}}
\end{array}\right)
$$

In Figure A.22 the output in the receiver location is plotted and the two curves represent the final computation done considering the off-diagonal as Cross PSDs of the signals and the one done considering the off-diagonal set to 0 . 


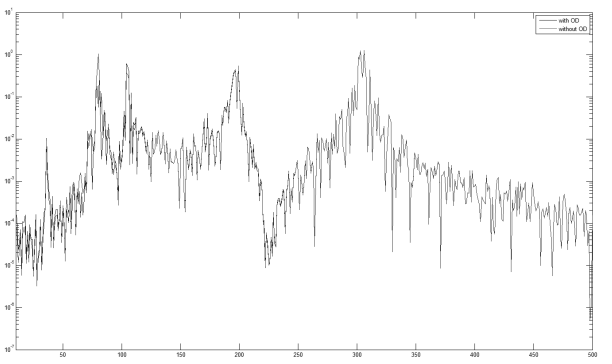

(a) $\varphi=0$

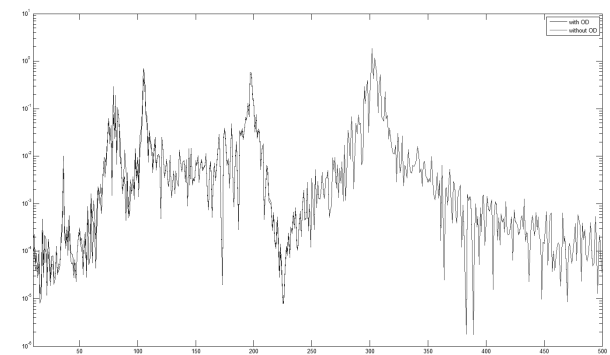

(c) $\varphi=\pi$

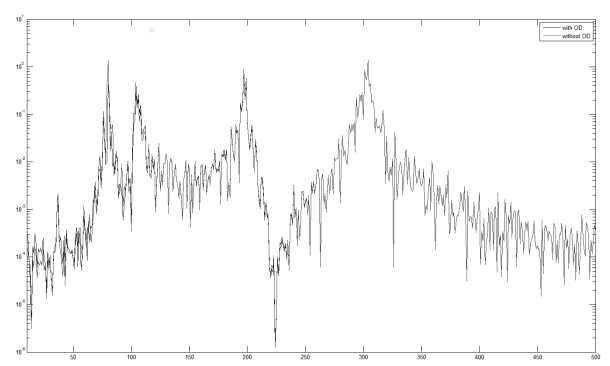

(b) $\varphi=\pi / 3$

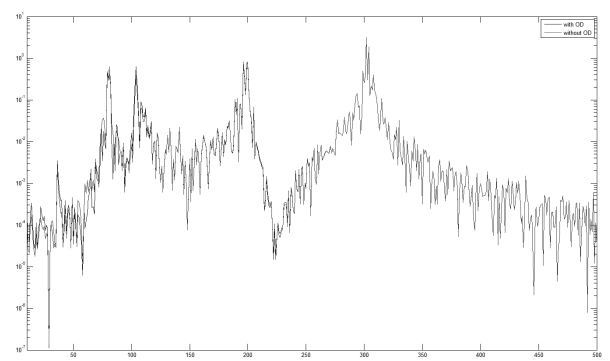

(d) $\varphi=4 / 3 \pi$

Figure A.22: Comparison considering off-diagonal terms as Cross PSDs or equal to 0 with the two signal defined by sines out of phase

As can be observed, there are slight differences only in the low-frequency range. Overall the difference is minimal. This is one of many examples run in this context and as a consequence the authors decided to use only the diagonal terms for the computations performed in this article. 
[1] O.C. Zienkiewicz and R.L. Taylor. The finite element method (third edition). McGraw-Hill Book Co., 1983.

[2] W. Desmet. Mid-frequency vibro-acoustic modeling: challenges and potential solutions. In P. Sas and B. Vanhal, editors, Proceedings of ISMA 2002: International Conference on Noise and Vibration, volume 1-5, pages 835-862, Leuven, Belgium, September 2002.

[3] M. Kompella and R. Bernhard. Measurement of the statistical variation of structural-acoustic characteristics of automotive vehicles. $S A E$ Technical Paper 931272, 1993.

[4] R.H. Lyon. Statistical Energy Analysis of Dynamical Systems: Theory and Applications. M.I.T. Press, Cambridge, 1975.

[5] R.H. Lyon and R.G. DeJong. Theory and Application of Statistical Energy Analysis, Second Edition. Butterworth-Heinemann, Boston, 1995.

[6] F.J. Fahy. Statistical energy analysis: a critical overview. Philosophical Transactions of the Royal Society of London, 346(1681):431-447, 1994.

[7] H.J. Hwang. Prediction and validation of high frequency vibration repsonses of nasa mars pathfinder spacecraft due to acoustic launch load using statistical energy analysis. In Institute of Environmental Sciences and Technology, ESTECH 2022 Conference, Anaheim, CA, USA, 2002.

[8] H.J. Hwang. Vibroacoustic analysis and experimental validation of the structural responses of nasa mars exploration rover spacecraft due to acoustic launch load. In Noise Conference 2003, Cleveland, OH, USA, 2003 .

[9] J.M. Larko and W.O. Hughes. Initial assessment of the ares i-x launch vehicle upper stage to vibroacoustic flight environments. In 14th International Congress on Sound Vibration, Cleveland, Ohio, 2008.

[10] G.S. Aglietti, S. Gabriel, R. Langley, and E. Rogers. A modeling technique for active control design studies with application to spacecraft microvibrations. The Journal of the Acoustical Society of America, 102(4):2158-66, 1997. 
[11] G.S. Aglietti, R.S. Langley, E. Rogers, and S.B. Gabriel. An efficient model of an equipment loaded panel for active control design studies. The Journal of the Acoustical Society of America, 108(4):1663-73, 2000.

[12] O. Takahara, N. Yoshida, H. Uchida, and K. Komatsu. Study of estimation method of microvibration with simulated satellite structure. In 59th International Astronautical Congress 2008, Glasgow, UK, 2008.

[13] V. Babuska, D.M. Coombs, J.C. Goodding, E.V. Ardelean, L.M. Robertson, and S.A. Lane. Modeling and experimental validation of space structures with wiring harnesses. Journal of Spacecraft and Rockets, 47(6):1038-1052, 2010.

[14] J.C. Goodding, E.V. Ardelean, V. Babuska, L.M. Robertson, and S.A. Lane. Experimental techniques and structural parameter estimation studies of spacecraft cables. Journal of Spacecraft and Rockets, 48(6):942-957, 2011.

[15] D.M. Coombs, J.C. Goodding, V. Babuska, E.V. Ardelean, L.M. Robertson, and S.A. Lan. Dynamic modeling and experimental validation of a cable-loaded panel. Journal of Spacecraft and Rockets, 48(6):958-974, 2011.

[16] W. Desmet, B. Pluymers, and O. Atak. "MID-FREQUENCY" - CAE Methodologies for Mid-Frequency Analysis in Vibrations and Acoustics. 2012 .

[17] P.J. Shorter and R.S. Langley. On the reciprocity relationship between direct field radiation and diffuse reverberant loading. Journal of the Acoustical Society of America, 117(1):85-95, 2005.

[18] P.J. Shorter and R.S. Langley. Vibro-acoustic analysis of complex systems. Journal of Sound and Vibration, 288(3):669-700, 2005.

[19] T. Huiban, Y. Baillion, and R. Valli. Prediction tool for micro-vibration sensitive payloads. In Aerospatiale Etablissement de Cannes, France, 1996.

[20] G. Stefanou. The stochastic finite element method: past, present and future. Computer Methods in Applied Mechanics and Engineering, 198(912):1031-1051, 2009. 
[21] M.C. Lin and Y.W. Kiang. Performance analysis of a radar target discrimination technique. IEEE Transactions on Aerospace and Electronic Systems, 28(2):363-369, 1991.

[22] S. Adhikari, M.I. Friswell, K. Lonkar, and A. Sarkar. Experimental case studies for uncertainty quantification in structural dynamics. Probabilistic Engineering Mechanics, 24(4):473-492, 2009.

[23] G.S. Aglietti, S.J.I. Walker, and A. Kiley. On the use of serep for satellite fem validation. Engineering Computations, 29:580-595, 2012.

[24] R.R. Craig and M.C.C. Bampton. Coupling of substructures for dynamic analyses. AIAA Journal, 6 (7):1313-1319, 1968.

[25] L. Hinke, F. Donhal, B.R. Mace, T.P. Waters, and N.S. Ferguson. Component mode synthesis as a framework for uncertainty analysis. Journal of Sound and Vibration, 324(1-2):161-178, 2009.

[26] D. Sarsri, L. Azrar, A. Jebbouri, and A. El Hami. Component mode synthesis and polynomial chaos expansions for stochastic frequency functions of large linear fe models. Computers 85 Structures, 89(3-4):346-356, 2011.

[27] H.J. Pradlwarter, G.I. Schueller, and G.S. Szekely. Random eigenvalue problems for large systems. Computers $\&$ Structures, 80(27-30):24152424, 2002.

[28] F. Dohnal, B.R. Mace, and N.S. Ferguson. Analysis of vibrations of systems with spatially correlated uncertainty in joints using a stochastic reduced basis method. In EURODYN 2008, 7th European Conference on Structural Dynamics, Southampton, UK, July 2008.

[29] M.P. Mignolet and C. Soize. Nonparametric stochastic modelling of structures with uncertain boundary conditions and uncertain coupling between substructures. In 49th AIAA/ASME/ASCE/AHS/ASC Structures, Structural Dynamics, and Materials Conference, Schaumburg (Chicago), Illinois, USA, April 2008.

[30] C. Soize and S. Mziou. Dynamic substructuring in the mediumfrequency range. AIAA Journal, 41(6):1113-1118, 2003. 
[31] M. Guedri, N. Bouhaddi, and R. Majed. Reduction of the stochastic finite element models using a robust dynamic condensation method. Journal of Sound and Vibration, 297(1-2):123-145, October 2006.

[32] M. Guedri, S. Ghanmi, R. Majed, and N. Bouhaddi. Robust tools for prediction of variability and optimization in structural dynamics. Mechanical Systems and Signal Processing, 23(4):1123-1133, May 2009.

[33] B. Van den Nieuwenhof and J-P. Coyette. Modal approaches for the stochastic finite element analysis of structures with material and geometric uncertainties. Computer Methods in Applied Mechanics and Engineering, 192(33-34):3705-3729, August 2003.

[34] L. E. Suarez and M. P. Singh. Dynamic condensation method for structural eigenvalue analysis. AIAA Journal, 30(4):1046-1054, April 1992.

[35] B. Mace and P. Shorter. A local modal/perturbational method for estimating frequency response statistics of built-up structures with uncertain properties. Journal of Sound and Vibration, 242(5):793-811, 2001.

[36] Z. Zhang, G. S. Aglietti, and W. Zhou. Microvibrations induced by a cantilevered wheel assembly with a soft-suspension system. AIAA Journal, 49(5):1067-1079, 2011.

[37] Z. Zhang, G. S. Aglietti, and W. J. Ren. Microvibration model development and validation of a cantilevered reaction wheel assembly. Applied Mechanics and Materials, 226:133-137, 2012.

[38] R.A. Masterson and D.W. Miller. Development and Validation of Empirical and Analytical Reaction Wheel Disturbance Models. Massachusetts Institute of Technology, 1999.

[39] R. A. Masterson, D.W. Miller, and R.L. Grogan. Development and validation of reaction wheel disturbance models: Empirical model. Journal of Sou, 249(3):575-598, 2002.

[40] K.C. Liu, P. Maghami, and C. Blaurock. Reaction wheel disturbance modeling, jitter analysis, and validation tests for solar dynamics observatory. In AIAA Guidance, Navigation and Control Conference and Exhibit, Honolulu, Hawaii, 2008. 
[41] P. C. Marucchi-Chierro and C. Galeazzi. The microvibration environment on artemis : A strategy for its characterization and control. In 46th International Astronautical Congress, Oslo, Norway, 1995.

[42] O.L. De Weck and D.W. Miller. Integrated modeling and dynamics simulation for the next generation space telescope. In Massachusetts Institute of Technology, 1999.

[43] L.M. Elias and D.W. Miller. A coupled disturbance analysis method using dynamic mass measurement techniques. In 43rd AIAA/ASME/ASCE/AHS/ASC Structures, Structural Dynamics, and Materials Conference, Denver, Colorado, 2002.

[44] L.M. Elias, F. Dekens, I. Basdogan, L. Sievers, and T. Neville. A methodology for modeling the mechanical interaction between a reaction wheel and a flexible structure. In Atronomical Telescopes and Instrumentation, Waikoloa, HI, USA, 2003.

[45] T. J. Young. Primer on the craig-bampton method (based on input from william b. halle). 2000. 